Kirsch- und Pflaumenkernöle, die fabrikmäßig aus Kirsch- und Pflaumensteinen nach dem von mir angegebenen Verfahren gewonnen worden sind, enthalten geringe Mengen Benzaldehyd und Benzoesäure $(0,00 \hat{6}-0,047 \%$, als Benzoesäure berechnet) und Spuren Blausäure (bis $6,5 \mathrm{mg}$ in $100 \mathrm{~g}$ ). Diese Bestandteile tun der Verwertung der Öle als Speiseöl keinen Abbruch. Der fremdartige Bittermandelgeschmack verliert sich bei der Aufbewahrung der Öle und kann durch Wasserdampf entfernt werden.

Die Kennzahlen der Öle waren folgende:

Kirschöl: 1. Spezifisches Gewicht 0,9218-0,9272, Mittel 0,9247. 2. Refraktometerzahl bei $25^{0}: 74,7-77,3$, Mittel 75,7. 3. Verseifungszahl: 192,1-197,8, Mittel 194,5. 4. Jodzahl nach v. Hübl: 111,6-122,6, Mittel 118,6.

Pfla umenkernöl: 1. Spezifisches Gewicht: 0,9193-0,9213, Mittel 0,9202. 2. Refraktometerzahl: $65,1-66,7$, Mittel 65,9 . 3. Verseifungszahl: $188,1-198,5$, Mittel 194,4. 4. Jodzahl: 103,6-121,1.

Die Refraktometerzahl der mit Äther extrahierten Öle aus gleichartigen Kernen bewegte sich bei den Pflaumen- und Zwetschensorten zwischen 63,5 und 67,2 und betrug im Mittel 65,1 , bei den Kirschen 75,2 und 81,6 , im Mittel 77,5 , bei den Pfirsichen 63,3 und 66,0, im Mittel 65,3, bei den Aprikosen 63,7 und 65,6, im Mittel 64,6. Den Ölkernen beigemengte Schalenteile geben beim Pressen an das Öl lösliche Stoffe ab, die den Säuregrad des Öles erhöhen und die Güte beeinträchtigen. Völlig schalenfreie Kerne geben feines, dem Mandelöl ähnliches Öl.

Die Preßkuchen von der Kirsch - und Pflaumenölgewinnung enthielten 22,81 bis $32,68 \%$ Rohprotein neben $9,16-21,29 \%$ Fett; sie stellen ein wertvolles Beifutter für Schweine dar. Von den Steinschalen völlig befreit und durch geeignete Behandlung mit Wasserdampf möglichst entbittert, können die Samen sowohl wie die Prefikuchen zu Back- und Konditorwaren Verwendung finden.

\title{
Bestimmung des Äthylalkohols bei Gegenwart flüchtiger Stoffe, insbesondere von Aldehyd und Aceton, und die gleich- zeitige Bestimmung der letzteren.
}

\author{
Von \\ Karl Hoopner.
}

Mitteilung aus dem Wissenschaftlichen Laboratorium der Königlichen Hauptlehranstalt für Zollbeamte in Berlin.

[Eingegangen am 27. September 1917.]

Die Mengenbestimmung des Äthylalkohols in alkoholhaltigen Erzeugnissen erfolgt fast ausschließlich und am genauesten aus der Dichte von Alkohol-WasserMischungen, wobei es von geringer Bedeutung ist, ob die Dichte mittels geeigneter Spindeln, mittels der Westphal'schen Wage oder mittels des Dichtefläschchens festgestellt wird. Voraussetzung ist nur, $\mathrm{da \beta}$ das alkoholhaltige Erzeugnis, dessen Dichte auf eine der angegebenen Weisen ermittelt werden soll, lediglich aus einer AlkoholWasser-Mischung besteht, demnach Extraktivstoffe und flüchtige Stoffe nicht oder doch nur in unerheblicher Menge enthält. Ist diese Voraussetzung nicht erfüllt, so müssen 
in jedem Falle die störenden nichtflüchtigen und flüchtigen Stoffe abgesondert und eine reine Alkohol-Wasser-Mischung hergestellt werden. Der im einzelnen einzuschlagende Weg richtet sich nach der Natur der die Dichte beeinflussenden Beimengungen. Bestehen letztere aus Extraktivstoffen, so lassen sie sich leicht durch Abtreiben vom Alkohol trennen, und die Bestimmung des Alkohols erfolgt durch Auffüllen des Abtriebes auf das ursprüngliche Maß oder Gewicht oder ein Vielfaches davon und durch Ermittelung der Dichte der so erhaltenen reinen Alkohol-WasserMischung. Das gleiche Verfahren unter Zusatz von Alkali oder Mineralsäure wird angewendet, wenn es sich um die Entfernung flüchtiger Säuren oder Basen handelt. Für alle übrigen Fälle, in denen das alkoholhaltige Erzeugnis neutrale flüchtige Stoffe, insbesondere künstliche und natürliche Ester, ätherische Öle u. a. enthält, sehen die Vorschriften der Branntweinsteuer-Ausführungsbestimmungen eine Reihe vorbereitender Maßnabmen vor, welche auf die Herstellung einer von störenden Beimengungen freien, reinen Alkohol-Wasser-Mischung hinzielen.

Nach der in Anlage 2 zu $\S 16$ Abs. 2 der Alkoholermittelungsordnung gegebenen Anleitung wird das alkoholhaltige Erzeugnis - Likör, Essenz u. a. - zur Entfernung vorhandener aromatischer Bestandteile in einer Schüttelbürette, nach dem Verdünnen mit Wasser, mit Kochsalz gesättigt und die Hälfte der gesättigten Kochsalzlösung abgetrieben; nach der in Anlage $21 \mathrm{zu} \$ 65 \mathrm{Abs} .2$ der BranntweinsteuerBefreiungsordnung gegebenen Anleitung werden die alkoholhaltigen Parfümerien, Kopf-, Zahn- und Mundwässer, deren Alkoholgehalt nicht nach Maßgabe der Alkoholermittelungsordnung festgestellt werden kann, mit Petroleumbenzin - bei Gegenwart von Harzen oder anderen Extraktivstoffen nach vorhergehendem Abtrieb - ausgeschüttelt; nach Ziffer III 88 der Anleitung für die Zollabfertigung (5. Nachtrag) endlich werden Fuselöle bei ihrer Prüfung auf einen 8 v. $H$. übersteigenden Alkoholgehalt mit Chlorcalciumlösung und Cumol geschüttelt und abgetrieben. In den nach diesen Vorschriften erhaltenen Alkohol-Wasser-Mischungen wird dann die Dichte auf eine der angegebenen Weisen ermittelt und der sich aus ihr ergebende Alkoholgehalt auf die angewendete Menge der Warenprobe bezogen.

Wenn auch in den meisten Fällen die amtlich vorgeschriebenen Verfahren zu brauchbaren Ergebnissen führen, so versagen sie doch bei einer nicht geringen Zahl der hierher gehörenden Erzeugnisse, Beispielsweise gelingt es nicht, in einer Rumessenz die künstlichen Ester in dem Maße vom Alkohol zu trennen, daf die Ermittelung des Alkoholgehaltes aus der Dichte ein zuverlässiges Ergebnis liefert. Was von den niederen Estern der niederen Fettsäuren - Ameisenäther, Essigäther -, welche einen wesentlichen Bestandteil der Rumessenzen bilden, gilt, trifft auch für andere flüchtige Stoffe wie Äthyläther, Chloroform, Benzol, Aldehyd, Aceton u. a. in gleicher Weise zu.

Diese Erfahrungen wie auch die dem Laboratorium gestellte Aufgabe, den Alkoholgehalt einer Alkohol-Aceton-Wasser-Mischung festzustellen, haben die Veranlassung $\mathrm{zu}$ diesbezüglichen Untersuchungen von Branntweingemischen verschiedenster Art gegeben und zu dem nachstehend beschriebenen Verfahren der Alkoholbestimmung und der gleichzeitigen Bestimmung ron Aceton und Aldehyd geführt.

Soweit die Bestimmung des Alkoholgehal tes in Frage kommt, gründet sich das Verfabren auf die leichte Oxydierbarkeit desselben zu Essigsäure mittels Kaliumbichromats und Schwefelsäure. Das bereits von Nicloux für die Bestimmung kleiner 
Alkoholmengen angegebene Verfahren hat bisher eine weitere Verbreitung nicht gefunden, weil, wie die Nachprüfungen anderer Bearbeiter ergeben haben, der vorhandene Alkohol bei zu schwacher Oxydation nur zu Aldehyd und bei zu starker Oxydation über die Essigsäure hinaus zu Kohlensäure oxydiert wird. Wenn auch die Möglich. keit ungenügender wie auch zu weit gehender Oxydation keineswegs bestritten werden soll, so können doch andererseits die Versuchsbedingungen - Konzentration, Versuchstemperatur und Einwirkungsdauer - so gewählt werden, daß die erwähnten, das ganze Verfahren sonst unhaltbar machenden Nachteile vollkommen ausgeschaltet werden, und daß das Chromsäureverfahren nicht nur zur Bestimmung geringer Mengen Alkohol, sondern auch jeder beliebigen Alkoholmenge anwendbar ist. Wie eingehende Untersuchungen bewiesen haben, werden unter bestimmten Bedingungen bei der Einwirkung von Kaliumbichromat und Schwefelsäure auf ein Gemisch von Alkohol, Aldehyd und Aceton die beiden ersteren vollständig zur Essigsäure oxydiert, während das schwerer oxydierbare Aceton unverändert bleibt.

Die Bestimmungen des Aldehyds und des Acetons beruhen auf der Umsetzung beider Stoffe mit Hydroxylaminchlorhydrat zu Aldoxim und Ketoxim unter Freiwerden von je einem Molekül Salzsäure.

Aus diesem Verhalten des Alkohols, Aldehyds und Acetons gegen Kaliumbichromat-Schwefelsäure einerseits und gegen Hydroxylaminchlorhydrat andererseits ergibt sich der folgende analytische Gang:

1. Alkohol und Aldehyd werden zu Essigsäure oxydiert; das unveränderte Aceton wird von der gebildeten Essigsäure durch Abtreiben getrennt, mittels Hydroxylaminchlorhydrat is das Ketoxim übergeführt und die freigewordene Salzsäure mit NormalLauge titriert:

$$
\left(\mathrm{CH}_{3}\right)_{2} \mathrm{CO}+\mathrm{NH}_{2} \cdot \mathrm{OH} \cdot \mathrm{HCl}=\left(\mathrm{CH}_{3}\right)_{2} \mathrm{C}: \mathrm{N} . \mathrm{OH}+\mathrm{H}_{2} \mathrm{O}+\mathrm{HCl} \text {. }
$$

2. Aldehyd und Aceton werden mittels Hydroxylaminchlorhydrat in das Aldoxim und das Ketoxim übergeführt, und die freigewordene Salzsäure wird mit Normal-Lauge titriert:

$$
\begin{gathered}
\mathrm{CH}_{3} \cdot \mathrm{OHO}+\left(\mathrm{CH}_{3}\right)_{2} \mathrm{CO}+2 \mathrm{NH}_{2} \cdot \mathrm{OH} \cdot \mathrm{HCl}=\mathrm{CH}_{3} \cdot \mathrm{CH}: \mathrm{N} \cdot \mathrm{OH}+\left(\mathrm{CH}_{3}\right)_{2} \mathrm{C}: \mathrm{N} \cdot \mathrm{OH} \\
+2 \mathrm{H}_{2} \mathrm{O}+2 \mathrm{HCl} .
\end{gathered}
$$

Aus dem Unterschiede der zu 1. und 2. verbrauchten Normal-Lauge ergibt sich der Gehalt an Aldehyd.

3. Alkohol und Aldehyd werden mit Kaliumbichromat-Schwefelsäure zu Essigsäure oxydiert, während Aceton unverändert bleibt:

$$
\begin{gathered}
3 \mathrm{CH}_{3} \cdot \mathrm{CH}_{2} \cdot \mathrm{OH}+3 \mathrm{CH}_{3} \cdot \mathrm{CHO}+3 \mathrm{~K}_{2} \mathrm{Cr}_{2} \mathrm{O}_{7}+12 \mathrm{H}_{2} \mathrm{SO}_{4}=6 \mathrm{CH}_{3} \cdot \mathrm{CO} \cdot \mathrm{OH} \\
+3 \mathrm{Cr}_{2}\left(\mathrm{SO}_{4}\right)_{3} \cdot \mathrm{K}_{2} \mathrm{SO}_{4}+15 \mathrm{H}_{2} \mathrm{O} .
\end{gathered}
$$

Aus dem Verbrauch an Kaliumbichromat berechnet sich unter Berücksichtigung des für die Oxydation des nach 2. ermittelten Alflehyds verbrauchten Kaliumbichromats der Gehalt an Alkohol.

\section{Ausfihrung der Untersuehung.}

A. Allgemeine Bemerkungen.

1. Enthält das zu untersuchende Erzeugnis auber Alkohol, Aldehyd, Aceton und Wasser flüchtige Säuren oder Basen oder leicht oxydierbare Extraktivstoffe, so sind dieselben durch Abtreiben, gegebenenfalls unter Zusatz von Alkali oder Mineralsäuren, zu entfernen; ein Überschuß von Alkali ist zur Verhütung einer Verharzung des Aldehyds zu vermeiden. 
2. Das zu untersuchende alkoholhaltige Erzengnis oder der aus ihm erbaltene Abtrieb ist für die Untersuchung soweit zu verdünnen, daß $100 \mathrm{ccm}$ nicht mehr als $4 \mathrm{~g}$ oder $5 \mathrm{ccm}$ Alkohol enthalten. Beträgt z. B. die Dichte der Mischung 0,857, entsprechend $66,1 \mathrm{~g}$ oder $82,6 \mathrm{~cm}$ Alkohol in $100 \mathrm{ccm}$, so sind zweckmäßig $25 \mathrm{ccm}$ der Mischung zu $500 \mathrm{ccm}$ zu verdünnen; beträgt die Dichte der Mischung 0,966, entsprechend $23,7 \mathrm{~g}$, oder $29,6 \mathrm{ccm}$ Alkohol in $100 \mathrm{ccm}$, so sind zweckmäßig $50 \mathrm{ccm}$ der Mischung zu $500 \mathrm{ccm}$ zu verdünnen; usw.

3. Mit Rücksicht auf die sich im Verhältnis zu der starken Verdünnung vervielfältigenden unvermeidlichen Versuchsfehler ist sorgfältiges Arbeiten und die Benutzung geeichter oder doch eichfähiger Meßgeräte (Kolben, Büretten, Pipetten) erforderlich. Beim Pipettieren wie auch beim Abtreiben sind die Spitzen der Pipetten und der Vorstöße in die vorgelegten Lösungen einzutauchen. Namentlich wegen der Flüchtigkeit des Aldehyds sind die Umsetzungsgefäße geschlossen zu halten und Umfüllungen nach Möglichkeit zu vermeiden. Auch ist darauf zu achten, daß die Ausgangs- und Versuchslösungen unter sich gleiche Wärmegrade aufweisen.

4. Da die doppeltnormale Kaliumbichromatlösung leicht auskrystallisiert, ist es, namentlich zur kühlen Jahreszeit, geboten, sich von der Abwesenheit fester Ausscheidungen zu überzeugen.

\section{B. Erforderliche Lösungen.}

1. Doppeltnormale Kaliumbichromatlösung, enthaltend $98,066 \mathrm{~g}$ im Liter,

2. Schwefelsäure, verdünnte, hergestellt aus $500 \mathrm{~g}$ oder $275 \mathrm{ccm}$ Schwefelsäure $(\mathrm{D}=1,84)$ und $500 \mathrm{ccm}$ Wasser,

3. Eisenvitriollösung, enthaltend $250 \mathrm{~g}$ Ferrosulfăt im Liter,

4. Natronlauge, enthaltend $100 \mathrm{~g}$ Natriumhydroxyd im Liter,

5. Hydroxylaminchlorhydratlösung, enthaltend $100 \mathrm{~g}$ im Liter,

6. Methylorangelösung, enthaltend $1 \mathrm{~g}$ im Liter,

7. Jodkaliumlösung, enthaltend $100 \mathrm{~g}$ im Liter und

8. Natriumthiosulfatlösung, annähernd 1/10-normal, enthaltend $25 \mathrm{~g}$ im Liter.

\section{Bestimmung des Acetons.}

$20 \mathrm{ccm}$ des zweckentsprechend verdünnten Gemisches oder Abtriebes läßt man in $50 \mathrm{ccm}$ doppeltnormale Kaliumbichromatlösung einfließen und fügt $100 \mathrm{ccm}$ verdünnte Schwefelsäure hinzu. Zur Vermeidung stärkerer Erwärmung wird der Kolben unmittelbar nach dem Säurezusatz unter der Wasserleitung gekühlt. Nach 2-3stündigem Stehen bei Zimmerwärme werden zur Reduktion der unveränderten Chromsäure $100 \mathrm{ccm}$ Eisenvitriollösung hinzugegeben. Unter Vorlage von $5 \mathrm{ccm}$ Natronlauge und $20 \mathrm{ccm}$ Wasser werden etwa $75 \mathrm{ccm}$ abgetrieben, und von diesem Abtriebe werden wiederum etwa $50 \mathrm{ccm}$ unter Vorlage von $20 \mathrm{ccm}$ Hydroxylaminchlorhydratlösung abgetrieben. Nach Verlauf ron 1 Stunde wird die bei der Unsetzung des Acetons mit dem Hydroxylaminchlorhydrat freigewordene Salzsäure unter Verwendung von 2 Tropfen Methylorangelösung mit Normallauge titriert. — Durch einen blinden Versuch ist der Verbrauch von $20 \mathrm{ccm}$ Hydroxylaminchlorhydratlösung an Normallauge zu bestimmen und in Abzug zu bringen. 


\section{Bestimmung des Aldehyds.}

$20 \mathrm{ccm}$ des zweckentsprechend verdünnten Gemisches oder Abtriebes läßt man in ein Gemisch von $20 \mathrm{~cm}$ Hydroxylaminchlorhydratlösung und $20 \mathrm{ccm}$ Wasser einfließen. Nach Verlauf von 1 Stunde wird wie vorher die freigewordene Salzsäure titriert und der bei dem blinden Versuch erhaltene Wert berücksichtigt.

Ist das verdünnte Gemisch oder der Abtrieb nicht neutral, so ist die zur Neutralisation erforderliche Säure- oder Alkalimenge ebenfalls in Anrechnung zu bringen.

Der Aldehydgebalt berechnet sich aus dem Ontersehiede zwischen dem ermittelten und gegebenenfalls berichtigten Verbrauche an Normallauge vor und nach der Oxydation.

\section{E. Bestimmung des Alkohols.}

$10 \mathrm{ccm}$ des zweckentsprechend verdünnten Gemisches oder Abtriebes läßt man in $25 \mathrm{ccm}$ doppeltnormale Kaliumbichromatlösung einfließen, setzt $50 \mathrm{~cm}$ verdünnte Schwefelsäure zu und küblt wie unter C. Nach 2-3-stündigen Stehen wird der Inhalt des Kolbens unter mehrmaligem Nachspülen in einen Meßkolben von $500 \mathrm{ccm}$ gebracht. Nach Auffüllen zur Marke und Durchmischen werden $50 \mathrm{ccm}$ in einen Jodkolben abpipettiert, mit etwa $20 \mathrm{ccm}$ Jodkaliumlösung versetzt und nach $10-15$ Minuten unter Zusatz von Wasser und unter Verwendung von Stärkelösung in bekannter Wejse mit Natriumthiosulfatlösung titriert.

Zur Feststellung des Wirkungswertes der Thiosulfatlösung dient ein blinder Versuch, indem $25 \mathrm{ccm}$ doppeltnormale Kaliumbichromatlösung und $50 \mathrm{cem}$ verdünnie Schwefelsäure in gleicher Weise zu $500 \mathrm{ccm}$ verdünnt und mit Thiosulfatlösung titriert werden.

Da der Gehalt an dem gleichzeitig mitbestimmten Aldehyd bereits nach $\mathrm{D}$ ermittelt ist, läßt sich unter Berücksichtigung des letzteren der Alkoholgehalt aus dem Verbrauch an Natriumthiosulfatlösung ohne weiteres berechnen.

F. Bestimmung geringer Aldehyd-und Acetonmengen.

Ist der Gehalt an Aldehyd und an Aceton im Vergleich zu dem Alkoholgehalte sehr gering, so werden bei der vorstehenden Arbeitsweise infolge der starken Verdünnung die zur titrimetrischen Bestimmung kommenden Aldehyd- und Acetonmengen so klein, daß das Untersuchungsergebnis unsicher werden kann. Dies trifft im allgemeinen z11, wenn $5 \mathrm{ccm}$ des neutralen unverdünnten Gemisches oder Abtriebes nach dem Einfließenlassen in $20 \mathrm{ccm}$ Hydroxylaminchlorhydratlösung weniger als $5 \mathrm{ccm}$ Normallauge zur Neutralisation verbrauchen. In einem solchen Falle werden $100 \mathrm{ccm}$ des unverdünnten Gemisches oder Abtriebes mit $100 \mathrm{ccm}$ gesättigter Chlorcalciumlösung versetzt und unter Benutzung eines geeigneten Aufsatzes abgetrieben, indem man den Vorstoß in etwa $5 \mathrm{ccm}$ Wasser eintauchen läßt, das sich in einem Misch. zylinder von $50 \mathrm{ccm}$ befindet. Von dem etwa $40 \mathrm{ccm}$ betragenden, mit $50 \mathrm{ccm}$ ge. sättigter Chlorcalciumlösung versetzten Abtrieb werden von neuem in gleicher Weise etwa $20 \mathrm{ccm}$ abgetrieben und in $5 \mathrm{ccm}$ Wasser aufgefangen. Schließlich werden auch von diesem Abtriebe, nach Zugabe von $50 \mathrm{cem}$ gesättigter Chlorcalciumlösung, 8 bis $10 \mathrm{ccm}$ abgetrieben und in etwa $15 \mathrm{ccm}$ Wasser aufgefangen. Der so erhaltene $\mathrm{Ab}$. trieb, welcher, wie bei der Berechnung der Untersuchungsergebnisse näher ausgeführt werden wird, neben Alkohol sämtlichen Aldehyd, jedoch nicht die Gesamtmenge des Acetons enthält, wird in einen Meßkolben von $500 \mathrm{ccm}$ übergeführt. Die Bestimmung des Aldehyds und Acetons erfolgt in der vorher beschriebenen Weise. 
Daß die Bestimmung des Alkohols in diesen Fällen nicht mit der angereicherten, sondern mit der ursprünglichen, aber zweckentsprechend verdünnten Probe auszuführen ist, versteht sich von selbst.

\section{G. Ergänzende Bemerkungen.}

Enthält das alkoholhaltige Erzeugnis nur Aldehyd oder nur Aceton, so vereinfacht sich das Untersuchungsverfabren naturgemäb erheblich.

Bei A bwesenheit von Aldehyd ergibt sich der Gehalt an Aceton unmittelbar aus der Titration der bei der Umsetzung mit Hydroxylaminchlorhydrat freiwerdenden Salzsäure und der Gehalt an Alkohol unmittelbar aus dem Verbrauch an Kaliumbichromat- bezw. Natriumthiosulfatlösung.

Bei $A b w e s e n$ beit von Aceton ergibt sich der Gehalt an Aldehyd ebenfalls unmittelbar aus dem Verbrauche an Normallauge, während bei der Bestimmung des Alkohols die dem ermittelten Aldehydgebalte entsprechende Chromsäuremenge zu berücksichtigen und von dem Gesamtverbrauche an Kaliumbichromat- bezw. Natriumthiosulfatlösung abzuziehen ist.

Bei der Bestimmung des Acetons und Aldehyds ist ein etwaiger Gehalt an Säuren oder Basen zu berücksichtigen.

\section{Berechnung des Alkohols, Aldehyds und Acetons.}

1. Aceton.

Aus der Gleichung:

$$
\left(\mathrm{CH}_{3}\right)_{2} \mathrm{CO}+\mathrm{NH}_{2} \cdot \mathrm{OH} \cdot \mathrm{HCl}=\left(\mathrm{CH}_{3}\right)_{2} \mathrm{C}: \mathrm{N} \cdot \mathrm{OH}+\mathrm{H}_{2} \mathrm{O}+\mathrm{HCl}
$$

folgt, daß $58,048 \mathrm{~g}$ Aceton $=36,47 \mathrm{~g}$ Salzsäure $=40,06 \mathrm{~g}$ Natriumbydroxyd oder $1000 \mathrm{ccm}$ Normal-Lauge sind.

Mithin ist 1 ccm Normal.Lauge $=0,058048 \mathrm{~g}$ Aceton.

2. Aldebyd:

a) Aus der Gleichung:

$\mathrm{CH}_{3} \cdot \mathrm{CHO}+\mathrm{NH}_{2} \cdot \mathrm{OH} \cdot \mathrm{HCl}=\mathrm{CH}_{3} \cdot \mathrm{CH}: \mathrm{N} \cdot \mathrm{OH}+\mathrm{H}_{2} \mathrm{O}+\mathrm{HCl}$

folgt, daß 44,032 $\mathrm{g}$ Aldehyd $=36,47 \mathrm{~g}$ Salzsäure $=40,06 \mathrm{~g}$ Natriumhydroxyd oder 1000 ccm Normal-Lange sind.

Mithin ist $1 \mathrm{ccm}$ Normal-Lauge $=0,044032 \mathrm{~g}$ Aldehyd.

b) Aus der Gleichung:

$3 \mathrm{CH}_{3} \cdot \mathrm{OHO}+\mathrm{K}_{2} \mathrm{Cr}_{2} \mathrm{O}_{7}+4 \mathrm{H}_{2} \mathrm{SO}_{4}=3 \mathrm{CH}_{3} \cdot \mathrm{CO} . \mathrm{OH}+\mathrm{Cr}_{2}\left(\mathrm{SO}_{4}\right)_{3} \cdot \mathrm{K}_{2} \mathrm{SO}_{4}+4 \mathrm{H}_{2} \mathrm{O}$ folgt, daß

$3.44,032 \mathrm{~g}$ Aldehyd $=294,2 \mathrm{~g}$ Kaliumbichromat,

$44,032 \mathrm{~g}$ Aldehyd $=98,066 \mathrm{~g}$ Kaliumbichromat oder $1000 \mathrm{cem} 2 \mathrm{~N}$.Kaliumbichromatlösung und

$2,2016 \mathrm{~g}$ Aldehyd $=4,9033 \mathrm{~g}$ Kaliumbichromat oder $1000 \mathrm{ccm} 1 / 10 \mathrm{~N}$.-Kaliumbichromatlösung sind.

Mithin ist $1 \mathrm{ecm} 1 / 10 \mathrm{~N}$ - Kaliumbichromat-bezw. Natriumthiosulfatlösung $=0,0022016 \mathrm{~g}$ Aldebyd und $1 \mathrm{~g}$ Aldehyd $=454,2 \mathrm{ccm} 1 / 10$ N.-Kaliumbichromat- bezw. Natriumthiosulfatlösung. 
3. Alkohol:

Aus der Gleichung:

$3 \mathrm{CH}_{3} \cdot \mathrm{CH}_{2} \cdot \mathrm{OH}+2 \mathrm{~K}_{2} \mathrm{Cr}_{2} \mathrm{O}_{7}+8 \mathrm{H}_{2} \mathrm{SO}_{4}=3 \mathrm{CH}_{3} \cdot \mathrm{CO} \cdot \mathrm{OH}+2 \mathrm{Cr}_{2}\left(\mathrm{SO}_{4}\right)_{3}$

folgt, $\mathrm{daB}$

$$
\text { . } \mathrm{K}_{2} \mathrm{SO}_{4}+11 \mathrm{H}_{2} \mathrm{O}
$$

$3.46,048 \mathrm{~g}$ Alkohol $=2.294,2 \mathrm{~g}$ Kaliumbichromat,

$23,024 \mathrm{~g}$ Alkohol $=98,066 \mathrm{~g}$ Kaliumbichromat oder $1000 \mathrm{ccm} 2$ N.-Kalium. bichromatlösung und

$1,1512 \mathrm{~g}$ Alkohol $=4,9033 \mathrm{~g}$ Kaliumbichromat oder $1000 \mathrm{cem} 1 / 10 \mathrm{~N}$. Kalium bichromat- bezw. Natriumthiosulfatlösung sind.

Mithin ist $1 \mathrm{ccm} 1 / 10$ N.-Kaliumbichromat- bezw. Natriumthiosulfatlösung $=0,0011512 \mathrm{~g}$ Alkohol.

\section{Beispiel.}

Die zu untersuchende neutrale Alkohol-Aldehyd-Aceton-Wasser-Mischung hatte bei $15^{\circ} \mathrm{C}$ die Dichte 0,853, entsprechend einem Alkoholgehalt von $67,2 \mathrm{~g}$ oder $84,0 \mathrm{ccm}$ in $100 \mathrm{ccm}$, und war demnach im Verhältnis $1: 20 \mathrm{zu}$ verdünnen.

Die Untersuchung der so verdünnten Lösung ergab:

Aceton: $20 \mathrm{ccm}$ der verdünnten Lösung (=1 ccm der ursprünglichen Mischung) verbrauchten nach erfolgter $\mathrm{Oxydation}$, Destillation und Umsetzung mit Hydroxylaminchlorhydratlösung 2,9 ccm Normal-Lauge. Da bei dem blinden Versuche zur Neutralisation von $20 \mathrm{ccm}$ Hydroxylaminchlorhydratlösung $0,2 \mathrm{~cm}$ Normallauge erforderlich waren, so berechnet sich der Acetongehalt auf

$$
(2,9-0,2) \cdot 0,058048 \cdot 100=15,67 \mathrm{~g} \text { in } 100 \mathrm{~cm} \text {. }
$$

Aldehyd: $20 \mathrm{cem}$ der verdünnten Lösung ( $=1 \mathrm{cem}$ der ursprünglichen Mischung) verbrauchten nach der Umsetzung mit Hydroxylaminchlorbydratlösung, abzüglich des blinden Versuches, $5,4-0,2=5,2 \mathrm{ccm}$ Normal-Lange. Da für das in der gleichen Lösungsmenge enthaltene Aceton 2,7 ccm Normal-Lauge erforderlich waren, so berechnet sich der Aldebydgehalt auf

$$
(5,2-2,7) \cdot 0,044032.100=11,01 \mathrm{~g} \text { in } 100 \mathrm{ccm} \text {. }
$$

Alkohol: $10 \mathrm{ccm}$ der verdünnten Lösung (=0,5 $\mathrm{cm}$ der ursprünglichen Mischung) wurden nach der Oxydation za $500 \mathrm{ccm}$ verdünnt, von denen $50 \mathrm{ccm}(=0,05 \mathrm{ccm}$ der nrsprünglichen Mischung) 49,6-26,5 =23,1 ccm Natriumthiosulfatlösung verbrauchten. Da 49,6 ccm Natriumthiosulfatlösung $50 \mathrm{ccm}{ }^{1} / 10 \mathrm{~N}$. Kaliumbichromatlösung, erhalten durch Verdüonen von $25 \mathrm{cem}$ $2 \mathrm{~N}$.-Kaliumbichromatlösung zu $500 \mathrm{ccm}$, entsprechen, so sind zur Oxydation des in $0,05 \mathrm{ccm}$ der ursprünglichen Mischung enthaltenen Alkohols und Aldehyds $\frac{23,10.50}{49,60}=23,30 \mathrm{~cm} \mathrm{1/10} \mathrm{N.-Ka.}$ linmbichromatlösung verbraueht worden.

Da ferner in $100 \mathrm{ccm}$ der ursprünglichen Mischung $11,01 \mathrm{~g}$ Aldehyd ermittelt worden sind, so waren in den zur Titration verwendeten $0,05 \mathrm{ccm}$ der ursprünglichen Mischung $\frac{0,05.11,01}{100}=0,0055 \mathrm{~g}$ Aldehyd enthalten, welche bei der Oxydation $0,0055.454,2=2,5 \mathrm{ccm}$ $1 / 10 \mathbf{N}_{\text {. }}$-Kaliumbichromatlösung verbraucht haben. Demnach waren zur Oxydation des in 0,05 com der ursprünglichen Mischung enthaltenen Alkohols $23,3-2,5=20,8 \mathrm{~cm}{ }^{1 / 10} \mathrm{~N}$.-Kalıumbichromatlôsung erforderlich.

Mithin waren in $100 \mathrm{ccm}$ der ursprünglichen Misehung $20,8 \cdot 0,0011512.2000=47,89 \mathrm{~g}$ Alkohol enthalten.

Geringer Aceton- und Aldehydgehalt. Bei Gemischen, in denen der Gehalt an Aceton und Aldehyd im Vergleiche zum Alkoholgehalt sehr gering ist, ist für die Ermittelung des Acetongehaltes folgendes zu beachten: 
Während die Trennung des bei $21^{\circ} \mathrm{C}$ siedenden Aldehyds von dem bei $78^{\circ} \mathrm{C}$ siedenden Alkohol nach der unter $\mathrm{F}$ gegebenen Vorschrift verhältnismäbig leicht gelingt, ist es nicht möglich, das bei $56^{\circ} \mathrm{C}$ siedende Aceton trotz der Verwendung gesättigter Chlorcalciumlösung durch fraktionierte Destillation so anzureichern, daß es vollständig in der letzten Fraktion ron 8-10 $\mathrm{cm}$ enthalten ist. Infolgedessen bleiben die für den Acetongehalt erhaltenen Werte erbeblich hinter den theoretischen zurück. In diesen Fällen wird der Acetongebalt in der Weise ermittelt, dafo der dem in dem Destillationsrückstande verbliebenen Acetonreste entsprechende Unterschied zwischen dem Laugenverbrauche der unverdünnten ursprünglichen Mischung und demjenigen der angereicherten verdünnten Lösung vor der Oxydation zu der nach der Oxydation verbrauchten Normal-Lauge hinzugerechnet wird.

Hat beispielsweise die bei der Umsetzung von $5 \mathrm{ccm}$ der ursprünglichen Mischung mit Hydroxylaminchlorhydratlösung freigewordene Salzsäure $3,0-0,2=2,8 \mathrm{ccm}$ Normal-Lauge und haben $25 \mathrm{ccm}$ der angereicherten und im Verhältnis von 100:500 $\mathrm{cm}$ verdünnten Lösung ( $=5 \mathrm{ccm}$ der ursprünglichen Mischung) vor der Oxydation $2,45-0,2=2,25 \mathrm{~cm}$ und nach der Oxydation $1,0-0,2=0,8 \mathrm{~cm}$ Normal-Lauge verbraucht, so berechnet sich der in $100 \mathrm{ccm}$ der ursprünglichen Mischung enthaltene

Aldehyd auf $(2,25-0,8), 0,044032.20=1,28 \mathrm{~g}$ und das

A ceton auf $[0,8+(2,8-2,25)] .0,058048.20=1,57 \mathrm{~g}$.

Nach dem vorstehend beschriebenen Verfahren sind zahlreiche Mischungen von Alkohol, Aldehyd, Aceton und Wasser versehiedenster Zusammensetzung untersucht worden. Die Ergebnisse waren, wie auch die von den Herren des Laboratoriums ausgeführten Untersuchungen ihnen in ihrer Zusammensetzung unbekannter Mischungen bestätigt haben, in allen Fällen befriedigend.

Die zu den angestellten Versuchen verwendeten Stoffe: Alkohol, Aldehyd und Aceton wurden zunächst nach dem Chromsäure- bezw. Hydroxylaminverfahren untersucht und lieferten die nachstehenden Ergebnisse:

Äthylalkohol, absoluter, $D_{15}=0,7949$.

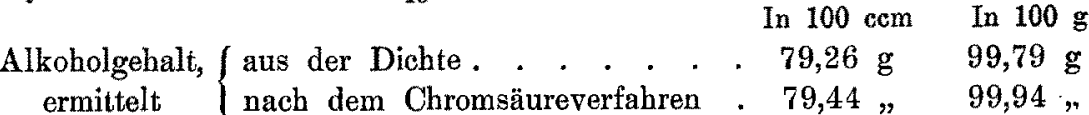

A cetaldehyd, Siedepunkt $21^{\circ} \mathrm{C}, \mathrm{D}_{\mathbf{1 5}}=0,7875$.

Aldehydgehalt, / nach dem Hydroxylaminverfahren 70,44 ” 88,95 " ermittelt $\{"$ "Chromsäureverfabren . 76,04 " 95,99 ,

A ceto $n, D_{15}=0,7988$.

Acetongehalt, ermittelt nach dem

Hydroxylaminverfahren . . . . . . . 77,08 " 96,96"

Bei diesen Ergebnissen der Prüfung der Ausgangsstoffe fällt namentlich auf, daß der Aldehydgehalt je nach dem angewendeten Verfahren verschieden gefunden wurde. Diese Erscheinung dürfte darauf zurückzuführen sein, daß der verwendete Acetaldehyd nicht chemisch rein war, sondern neben 89 Gewichtsteilen v. H. Aldehyd noch andere durch Chromsäure oxydierbare Stoffe enthielt.

Aus diesen Ausgangsstoffen wurden die Versuchslösungen in der Weise hergestellt, daß in gewogene Meßkolben von $100 \mathrm{ccm}$ abgemessene Mengen Alkohol, 
Aldehyd und Aceton eingewogen, die Kolben nach Zusatz von Wasser gekühlt und schließlich bei Zimmerwärme mit Wasser zur Marke aufgefüllt wurden. Hierbei war namentlich beim Aldehydzusatz sowohl die starke Wärmeentwickelung als auch die starke Zusammenziehung der Mischung beachtenswert.

Aus der großen Zahl der angestellten Versuche seien nur einige wenige in der folgenden Zusammenstellung, aus welcher sowohl die Zusammensetzung als auch die einzelnen Untersuchungsergebnisse ersichtlich sind, mitgeteilt.

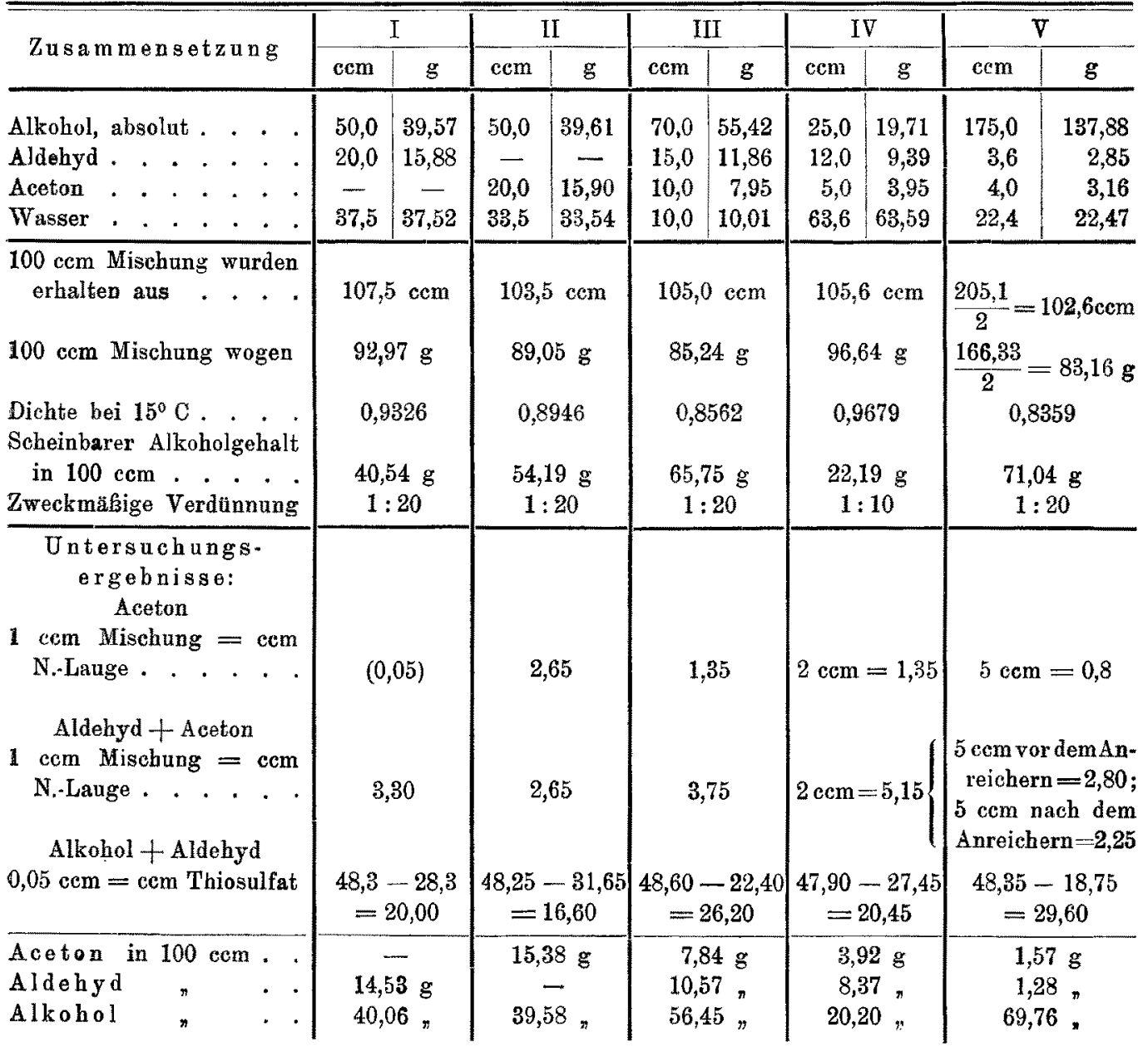

Nach diesen Ergebnissen decken sich die analytisch ermittelten Werte nicht völlig mit den zugesetzten Mengen der einzelnen Ausgangsstoffe, und zwar ist im allgemeinen der Aceton- und besonders der Aldehydgehalt zu niedrig und der Alkoholgehalt zu hoch ausgefallen. Diese Abweichungen der gefundenen von den theoretischen Werten sind indessen, wie bereits bemerkt worden ist, vornehmlich darauf zurückzuführen, daß einerseits das verwendete Aceton nur 97 Gewichtsteile v. H. Aceton und der verwendete Aldehyd sogar nur 89 Gewichtsteile v. H. Aldehyd nach 
dem Hydroxylaminverfabren, und dalo andererseits letzterer nach dem Chromsäureverfahren 96 Gewichtsteile v. H. Aldehyd enthalten hat. Infolgedessen mußten die für Aceton und Aldehyd ermittelten Werte unterhalb der zugesetzten Menge bleiben, wäbrend die Alkoholbestimmung bei Gegenwart von Aldehyd wegen der in dem verwendeten Aldehyd enthaltenen fremden, die Chromsäure gleichfalls reduzierenden Stoffe zu hohe Werte ergeben mubte.

In gleicher Weise wie der Äthylalkohol werden auch die Homologen des Äthylalkohols von dem Chromsäuregemisch unter den angegebenen Versuchsbedingungen zu den Fettsäuren mit gleicher Kohlenstoffzahl oxydiert. Daher ist es, wie auch die Versuche bestätigt haben, nicht möglich, in Branntweinen und anderen alkoholhaltigen Erzeugnissen, welche das niedrigste Glied der einwertigen Alkohole, den Methylalkohol, oder welche die den wesentlichen Bestandteil der Fuselöle bildenden höheren Glieder dieser Reihe, den Propyl-, den Butyl- und den Amylalkohol, enthalten, den Äthylalkohol nach dem Chromsäureverfahren zu bestimmen. Bei einem erheblicheren Fuselölgehalte wird die Alkoholstärke am zweckmäßigsten aus der Dichte bestimmt und von dem so ermittelten scheinbaren Alkoholgehalte der nach dem Röse'schen Verfahren gefundene Gehalt an Fuselöl, dessen Dichte von derjenigen des Äthylalkohols nicht sehr verschieden ist, abgezogen. Im allgemeinen ist jedoch der Fuselölgehalt der in Frage kommenden Erzeugnisse so gering, daß er vernachlässigt werden kaun. Aber auch in diesen Fällen bietet das Chromsäureverfahren gegenüber der Bestimmung des Alkohols aus der Dichte keine merklichen Vorteile.

Erheblich günstiger als bei den fuselölhaltigen Branntweinen liegen die Verhältnisse für das Chromsäureverfahren bei den alkoholhaltigen Erzeugnissen, welche andere neutrale flüchtige Stoffe wie Benzol, Chloroform, einfache und zusammengesetzte Äther, Ester der Fettsäuren und aromatischen Säuren und ätherische Öle enthalten, und welche namentlich bei ihrer Ausfuhr gegen Steuervergütung häufiger Gegenstand der Untersuchung, insbesondere der Feststellung des Gebaltes an vergütungsfähigem Alkohol werden.

Die genannten und äbnliche, die zuverlässige Ermittelung des Alkoholgehaltes aus der Dichte ausschließende Beimengungen lassen sich nach ihrer Oxydierbarkeit und nach ihrer Wasserlöslichkeit in drei Gruppen sondern:

Sind die neutralen flüchtigen Beimengungen verbältnismäbig sobwer oxyd i erbar, werden sie also unter den angegebenen Versucbsbedingungen von Chromsäure nicht angegriffen, so ist es belanglos, ob sie bei der vorzunehmenden starken Verdünnung gelöst bleiben oder abgeschieden werden. In diese Gruppe der schwer oxydierbaren neutralen flüchtigen Stoffe gehören z. B. Chloroform und Benzol, von deuen bei der Verdünnung 1:20 das erstere gelöst bleibt, das letztere dagegen ausgeschieden wird und leicht durch Filtration von dem schwachen Branntwein getrennt werden kaun.

Von den verhältnismäßig leicht ox ydierbaren Beimengungen lösen sich die niederen Glieder der einfachen und zusammengesetzten Äther und der Fettsäureester ziemlich leicht in Wasser, während die höheren Glieder dieser Reihen, ferner die Ester der aromatischen Säuren und die ätherischen Öle nur schwer in Wasser löslich sind. Infolgedessen werden die zur letzten Gruppe gehörenden, in Wasser schwer löslichen neutralen flüchtigen Stoffe bei der starken Verdünnung ohne weiteres ausgeschieden und können leicht durch Filtration der Einwirkung der Chromsäure entzogen werden. Dem Übelstande, daß bei der starken Verdünnung namentlich die ätherischen Ole häufig schwer auflösbare Emulsionen bilden, läßt sich in einfacher Weise dadurch 
begegnen, daf man der verdünnten Lösung ein auf Chromsäure nicht einwirkendes anorganisches Salz, am besten Natriumsulfat, zusetzt oder das alkoholhaltige Erzeugnis in Natriumsulfatlösung einfließen läßt und darauf die Salzlösung mit Petroleumbenzin ausschüttelt. - In gleicher Weise gelingt es auch, die Glieder der zweiten Gruppe, die verhältnismäßig leicht oxydierbaren und in Wasser löslichen niederen Äther und Fetisäureester von dem verdünnten Alkohol zu trennen.

Versuche, auch die Fuselöle mittels Natriumsulfats und Petroleumbenzins vom Branntwein zu scheiden, haben ein befriedigendes Ergebnis nicht gehabt. Vielmehr hat es sich gezeigt, daß die Fuselöle nur zum geringeren Teile in das Petroleumbenzin übergehen und zum größeren Teile in dem verdünnten, stark natriumsulfathaltigen Branntwein gelöst bleiben. Dieses Verhalten ist wohl daraus zu erklären, daß der noch in jedem Verhältnis mit Wasser mischbare Propylalkohol auch in schwach alkoholhaltiger Natriumsulfatlösung löslich ist, daß dagegen die Löslichkeit der folgenden einwertigen Alkohole in Wasser bezw. Natriumsulfatlösung mit steigendem Kohlenstoffgehait sinkt und umgekehrt die Löslichkeit in anderen Lösungsmitteln wie Petroleumbenzin steigt.

Demnach empfiehlt es sich, für die neutrale flüchtige Stoffe enthaltenden alkoholhaltigen Erzeugnisse - mit Ausnahme derjenigen, welche beachtenswerte Mengen Methylalkohol oder Fuselöl enthalten - die Abscheidung der störenden Beimengungen, mögen diese schwer oder leicht oxydierbar und schwer oder leicht in Wasser löslich sein, mittels Natriumsulfats und Petroleumbenzins allgemein vorzuschreiben.

Ein in allen Fällen zum Ziele führendes Verfahren ist das folgende:

Je nach der scheinbaren Alkoholstärke des alkoholhaltigen Erzeugnisses oder des aus ihm erhaltenen Abtriebes läßt man 25 oder $50 \mathrm{~cm}$ in etwa $400 \mathrm{ccm}$ Natrium. sulfatlösung, welche $150 \mathrm{~g}$ wasserfreies Salz im Liter enthält, einfließen, füllt mit der Natriumsulfatlösung auf $500 \mathrm{ccm}$ auf, führt den Inhalt des Meßkolbens in einen Scheidetrichter über und schüttelt mit $50 \mathrm{ccm}$ Petroleumbenzin ans. Bei in Wasser verhältnismäßig leicht löslichen niederen Äthern und Fettsäureestern und ähnlichen Stoffen ist es zweckmäbig, das Ausschütteln mit der gleichen Menge Petroleumbenzin zu wiederholen. Wenn sich die Schichten vollkommen getrennt haben, am besten am folgenden Tage, wird die völlig klare Salzlösung - gegebenenfalls unter Verwendung eines trocknen Filters - abgelassen. In $10 \mathrm{ccm}$ der verdünnten Lösung $(=0,50$ bezw. 1,0 cem der ursprünglichen Probe oder des Abtriebes) wird der Alkoholgehalt mittels Chromsäure in der beschriebenen Weise bestimmt.

Gegenüber der Verbindung des Aussalzens mit dem Ausäthern bietet das in den Branntweinsteuer-Ausführungsbestimmungen vorgeschriebene Aussalzen oder Ausäthern den Nachteil, daß bei alkoholreichen Branntweinen und anderen alkoholhaltigen Erzeugnissen die Abscheidung der störenden flüchtigen Bestandteile bäufig nur eine unvollkommene ist, oder daf das Lösungsmittel zum geringen Teil in den Branntwein übergeht. Im letzten Falle wird die Alkoholbestimmung aus der Dichte infolge ihrer Beeinflussung durch das spezifisch leichte Petroleumbenzin stets zu hohe Werte liefern, während im ersten Falle je nach der Dichte der flüchtigen Bestandteile die Alkòolbestimmung $\mathrm{zu}$ hohe wie $\mathrm{zu}$ niedrige wie auch zuweilen richtige Ergebnisse liefern wird. Wenn auch durch Verbindung der beiden amtlich vorgeschriebenen vorbereitenden Maßnahmen, des Aussalzens und des Ausätherns, die Abscheidung der störenden flüchtigen Stoffe gesteigert und die Löslichkeit des Petroleumbenzins herab. gesetzt werden kann, so bleibt doch zwischen der Alkoholbestimmung aus der Dichte 
und derjenigen nach dem Chromsäureverfahren der grundlegende Unterschied bestehen, daß bei der ersteren stärkere Verdünnungen nach Möglichkeit zu vermeiden sind, daß dagegen die letztere starke Verdünnungen - unter 5 Raumteile v. H. Alkohol - zur Voraussetzung hat. Es unterliegt keinem Zweifel, und es bedarf kaum eines experimentellen Beweises, daß alkoholreiche Branntweine trotz Aussalzens oder Ausätherns oder der Verbindung beider Maßnahmen erheblich größere Mengen an störenden flüchtigen Stoffen, namentlich an niederen Äthern und Fettsäureestern, in Lösung halten werden als alkoholarmé. In erster Linie in der starken Verdünnung und erst in zweiter Linie in 'der Verbindung des Aussalzens und Ausătherns liegt der unbestreitbare Vorzug des Chromsäureverfahrens zur Bestimmung des Alkohols in derartigen, neutrale flüchtige Stoffe enthaltenden Branntweinen gegenüber der Bestimmung aus der Dichte. Dazu kommt noch, daß die Alkoholbestimmung nach dem Chromsäureverfahren in allen Fällen auf kaltem Wege ausgeführt wird, daß dagegen der Alkoholbestimmung aus der Dichte im Falle erfolgten Aussalzens ein Abtrieb des Branntweins vorauszugehen hat.

Von einer eingehenden planmäBigen experimentellen Beweisführung zur Erhärtung der vorstehenden Ausführungen muß vorläufig, hauptsächlich infolge der derzeitigen Unmöglichkeit der Beschaffung von Petroleumbenzin, abgesehen werden. Gleichwohl seien nachstehend (S. 465 und 466) einige hierher gehörende Untersuchungen mitgeteilt, aus welchen einerseits der Einfluß des Aussalzens und Ausätherns auf die Alkoholermittelung nach dem Chromsäureverfahren und andererseits die Abweichungen der vor wie auch nach der Vorbehandlung mit Natriumsulfat und Petroleumbenzin erhaltenen Alkoholwerte von den wahren Alkoholstärken ohne weiteres ersichtlich sind.

Die aus den vorstehenden Ausführungen und aus den bisher durchgeführten Untersuchungen sich ergebenden Schlußfolgerungen lassen sich kurz, wie folgt, zusammenfassen:

Das Chromsäureverfahren soll das bisher allgemein übliche Verfahren der Alkoholbestimmung aus der Dichte nicht bei solchen Branntweinen und anderen alkoholhaltigen Erzeugnissen ersetzen, bei denen es durch Abtreiben, mit oder ohne Zusatz von Alkalien und Mineralsäuren, gelingt, die die Dichte beeinflussenden Stoffe auszuschalten und einen lediglich aus Alkohol und Wasser bestehenden Abtrieb zu erhalten, ferner auch nicht in denjenigen Fällen, in denen die neutralen flüchtigen Stoffe aus einwertigen Alkoholen der Fettreihe, insbesondere aus Methylalkohol und Fuselöl, bestehen. Dagegen ist dem Chromsäureverfahren dann der Vorzug zu geben, wenn die alkoholhaltigen Erzeugnisse andere flüchtige Beimengungen wie Aldehyd, Aceton, Chloroform, Benzol, einfache und zusammengesetzte Äther, Ester der Fettsäuren und aromatische Säuren, ätherische Öle oder ähnliche Stoffe enthalten, deren Abscheidung nach einem der amtlich vorgeschriebenen Verfahren nicht in einem solchen Grade möglich ist, daß die Alkoholbestimmung aus der Dichte zuverlässige Ergebnisse liefert.

Uberdies ermöglicht das Chromsäureverfahren bei aldehyd- und acetonhaltigen Branntweinen und anderen alkoholhaltigen Erzeugnissen nicht nur die Bestimmung ihres Alkoholgehaltes, sondern auch eine mit letzterer in einfacher Weise zu verbindende Bestimmung ihres Aldehyd- und Acetongehaltes. 


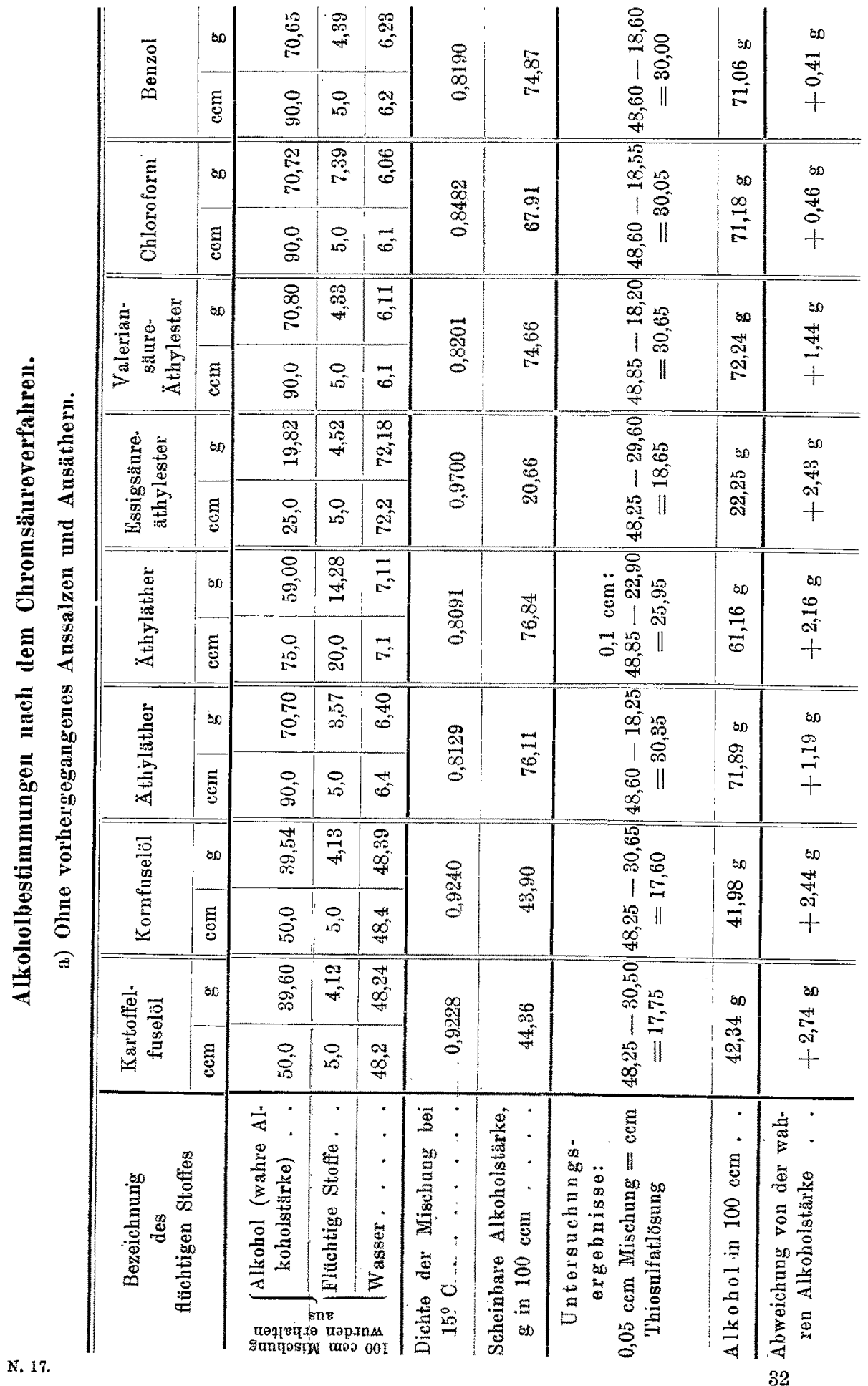




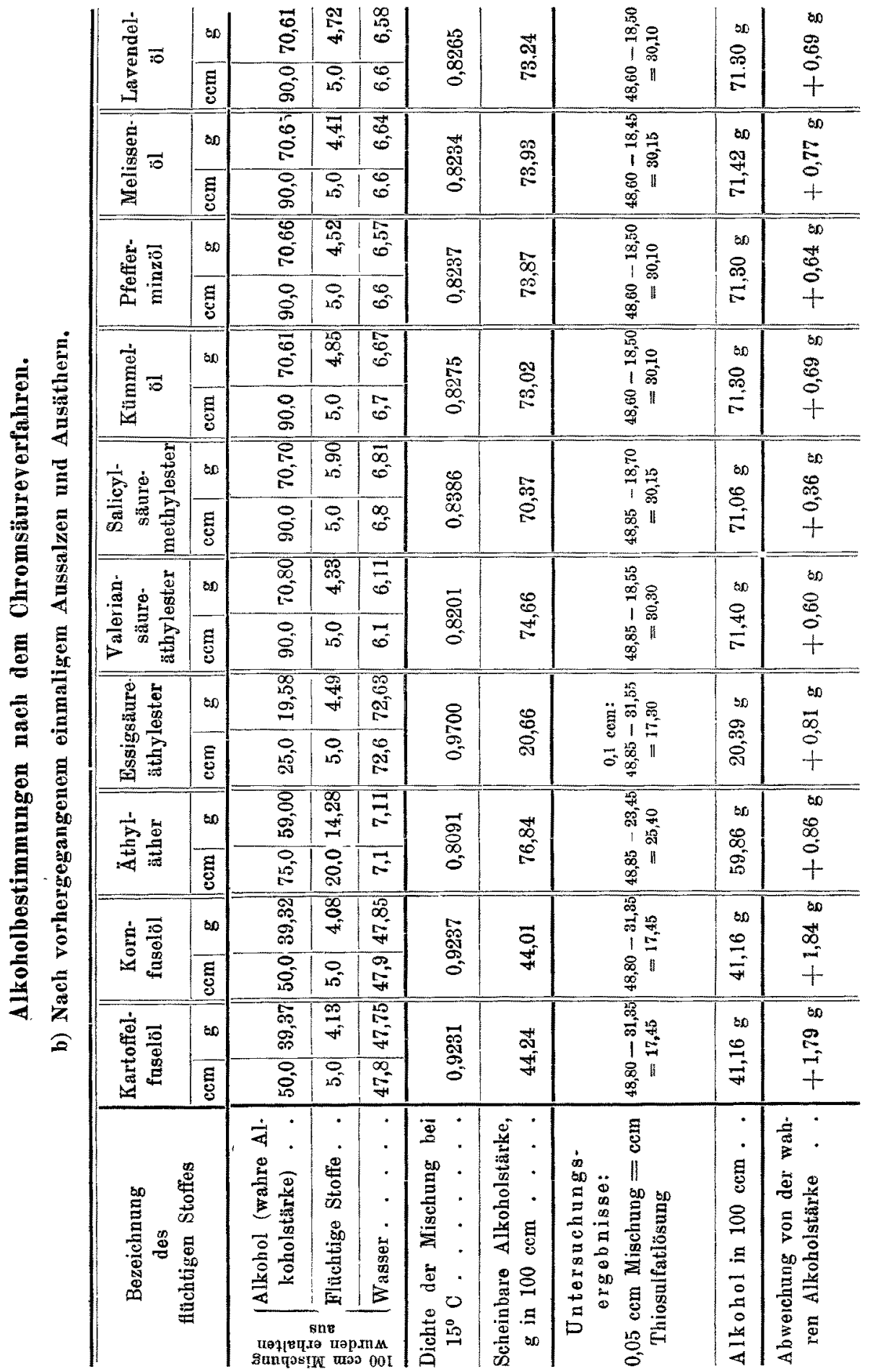

